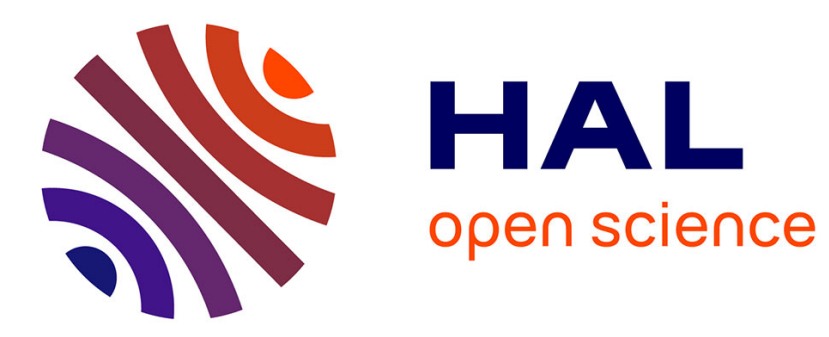

\title{
Référentialité et découpage des savoirs dans l'enseignement professionnel agricole
}

Laurent Fauré, Cécile Gardiès, Jean-François Marcel

\section{To cite this version:}

Laurent Fauré, Cécile Gardiès, Jean-François Marcel. Référentialité et découpage des savoirs dans l'enseignement professionnel agricole. Spirale - Revue de Recherches en Éducation , 2017. hal01753398

\section{HAL Id: hal-01753398 \\ https://hal.science/hal-01753398}

Submitted on 29 Mar 2018

HAL is a multi-disciplinary open access archive for the deposit and dissemination of scientific research documents, whether they are published or not. The documents may come from teaching and research institutions in France or abroad, or from public or private research centers.
L'archive ouverte pluridisciplinaire HAL, est destinée au dépôt et à la diffusion de documents scientifiques de niveau recherche, publiés ou non, émanant des établissements d'enseignement et de recherche français ou étrangers, des laboratoires publics ou privés. 


\title{
REFERENTIALITE ET DECOUPAGE DES SAVOIRS DANS L'ENSEIGNEMENT PROFESSIONNEL AGRICOLE
}

\author{
LE CAS DE L'INFORMATION-DOCUMENTATION \\ ET DES SCIENCES ET TECHNIQUES DES AGROEQUIPE- \\ MENTS
}

\begin{abstract}
Résumé : La question des savoirs dans un enseignement professionnel se pose à plusieurs niveaux et notamment en ce qui concerne la référentialité des savoirs à enseigner et du rapport à ces savoirs par les différents acteurs de la communauté éducative. L'enseignement agricole est «original» dans le paysage des formations en France du fait de la manière dont les savoirs sont découpés. Dès lors, nous analysons l'impact de ce découpage particulier des savoirs pour les enseignants. Nous étudions les liens entre savoirs scolaires tels que définis par l'institution et la référence de ces savoirs pour les enseignants à partir d'un cadre théorique articulant leur épistémologie scolaire et leur épistémologie professionnelle et en mobilisant une démarche méthodologique qualitative dont nous analysons et discutons les résultats au regard des pratiques d'enseignement.

Mots-clefs : Enseignement agricole, épistémologie scolaire, épistémologie pro-
\end{abstract} fessionnelle, référentiel de formation, savoirs de référence.

\section{INTRODUCTION ET PROBLEMATIQUE}

La question des savoirs dans un enseignement professionnel se pose à plusieurs niveaux et notamment celui de la référentialité des savoirs à enseigner et du rapport à ces savoirs par les différents acteurs de la communauté éducative. Ainsi, dans le champ de la didactique, si nous considérons que la discipline scolaire a pour finalité de «fabriquer de l'enseignable» (Chervel, 1988: 90) à partir de disciplines académiques ou de pratiques sociales de référence via un processus de «transposition didactique » (Chevallard, 1985/1991 ; Martinand, 1989), nous admettons que chaque discipline désigne un domaine scolaire d'études. Le champ scientifique de l'éducation précise même que «les disciplines n'apparaissent que quand elles sont constituées en ensembles cohérents de concepts, de notions, et de méthodologies qui leur sont propres » (Arenilla, Gossot \& Rolland, 1996 : 81-81). Toute division du savoir conduit inévitablement à une fragmentation disciplinaire.

La notion de discipline recoupe donc celle de connaissances et de savoirs limités à sa spécificité. Cependant les disciplines peuvent évoluer en fonction de l'avancement des connaissances de la science de référence et en fonction des significations portées par la conscience collective. "Les logiques disciplinaires visent fondamentalement à l'institutionnalisation et à la normalisation des discours et des pratiques [...] les logiques scientifiques ambitionnent la construction de

$$
\text { Spirale - Revue de Recherches en Éducation - } 2016 N^{\circ} 59 \text { (- }
$$


nouvelles connaissances » (Jeanneret \& Ollivier, 2004 :13). Dans l'enseignement professionnel l'ensemble des disciplines semble confronté à la définition de son champ de savoirs et à la prégnance de la visée professionnelle. Dans le paysage des formations en France, le pilotage institutionnel de l'enseignement agricole par le ministère de l'agriculture, et non celui de l'éducation nationale, marque son originalité. Une des spécificités de cette filière est la manière dont les savoirs sont regroupés. L'enseignement y est en effet modulaire et c'est autour de problématiques que les disciplines sont regroupées. Cette organisation modulaire permet ainsi de nouvelles connexions institutionnelles entre disciplines, qui vont jusqu'à des espaces d'interdisciplinarité (Gardiès \& Hervé, 2015). Or, « enseigner consiste à donner une certaine intelligibilité des savoirs » (Margolinas, 2014 : 20), ce qui interroge à notre sens le rapport des enseignants à l'épistémologie de leur discipline. Quel est alors l'impact de ce découpage particulier des savoirs dans l'enseignement agricole pour les enseignants ? Comment, à partir de ce découpage particulier, se construisent les savoirs enseignés, à partir de quelles références ? Comment se construit l'épistémologie professionnelle des enseignants lorsqu'ils sont confrontés à des champs disciplinaires multiples ?

Dès lors, nous proposons dans cet article d'étudier les liens entre savoirs scolaires tels que définis par l'institution enseignement agricole et la question de la référence de ces savoirs pour les enseignants à partir d'un cadre théorique articulant leur épistémologie scolaire et professionnelle et en mobilisant une démarche méthodologique qualitative. Les éléments empiriques concernent les préparations communes (entre deux enseignants) de séances pédagogiques. Celles-ci (au nombre de quatre) ont été filmées. Les séances étudiées se situaient dans deux disciplines scolaires : information-documentation d'une part et Sciences et techniques des agroéquipements (STEA) d'autre part. Ces deux disciplines ont la particularité d'être spécifiques à l'enseignement agricole. Définies relativement récemment, elles ont en commun un choix de savoirs à enseigner dont la référence n'est pas évidente a priori. L'objectif ici est de catégoriser les savoirs autour de ceux prescrits par les référentiels, puis mis en débat dans la phase de préparation des séances pédagogiques par les enseignants pour approcher la figure du «professionnel visé » par l'enseignement professionnel agricole. Nous analysons ainsi les données recueillies pour discuter le découpage et la référentialité des savoirs dans l'enseignement agricole et comment cela interroge les pratiques d'enseignement.

\section{CADRE THEORIQUE}

D'un point de vue théorique, et pour comprendre les enjeux des savoirs à dispenser pour les enseignants, nous proposons de voir ce que recouvre l'épistémologie scolaire. Celle-ci peut être entendue au sens d'une posture largement partagée dans la discipline scolaire et constitutive du corps de savoirs de référence qui fonde les contenus d'enseignement. Les savoirs à enseigner proviennent d'une extraction de corpus de savoirs produits dans les établissements d'enseignement supérieur et de recherche; ces savoirs savants constituent un cadre de référence commun normalement partagé. En effet, les savoirs en tant que domaines recensés, catalogués sont produits dans un contexte historique et social, ils font référence à des cultures. Chevallard, chercheur en didactique des mathématiques, a développé, dans les années quatre-vingt, des travaux autour de la transposition didactique. «Au sens restreint, la transposition didactique désigne donc le passage du savoir savant au savoir enseigné. Or c'est à la confrontation de ces deux ter- 
mes, à la distance qui les sépare, par-delà ce qui les rapproche et impose de les confronter, que l'on peut le mieux saisir la spécificité du traitement didactique du savoir »(Chevallard, 1985/1991:20).

La transposition didactique va donc consister à remettre en forme le savoir de référence, à le «traduire » et non à le simplifier. Cette traduction didactique permet de recréer ce savoir en situation d'enseignement, situation différente de celle de construction du savoir de la recherche scientifique. Les savoirs ne sont pas isolés mais les rapports personnels ou institutionnels aux savoirs sont en relation avec la tâche pour laquelle ces savoirs sont mobilisés, comme le souligne Chevallard «nul savoir ne saurait s'autoriser de lui-même » (Chevallard, 1996 : 146). Pour lui, « l'enseignement d'un savoir est toujours la réalisation d'un projet social » (Chevallard, 1985/1991: 148) dans le sens où le savoir possède un habitat et y occupe une fonction, ce qui implique une multilocation institutionnelle.

Le statut du savoir enseigné se définit en relation avec sa référence puisque tout contenu doit faire référence à un savoir ou à une pratique reconnus comme légitimement enseignable par la société (Arsac, Chevallard, Martinand, \& Tiberghien, 1994). Autrement dit, le savoir à enseigner dans une discipline doit reposer sur une reconnaissance du savoir savant et/ou des pratiques qui peuvent être associées au savoir savant, sociales, culturelles mais aussi les pratiques professionnelles (savoirs en acte). Mais ce savoir à enseigner, au-delà de sa légitimité, doit aussi être pertinent et ne peut être considéré comme un processus achevé mais plutôt comme processus en cours de stabilisation ou en cours de constitution. Certains savoirs peuvent être parés d'une légitimité aux dépens des autres car les savoirs sont constitués par leur socialisation et leur légitimité (Beillerot, BlanchardLaville, Mosconi, 1996).

La pertinence des savoirs enseignés, évaluée par rapport aux compétences visées, peut entraîner un certain amalgame dans l'identification des savoirs à enseigner dans la mesure où leur référentialité peut être multiple. Or, les contenus des connaissances individuelles sont souvent implicites et n'impliquent pas toujours un savoir partagé au sein de pratiques pourtant communes. Losfeld (1990: 163) souligne l'importance de l'individualisation de la connaissance, puisque «toute connaissance ne peut que prendre appui sur ce qui a été fait ou dit auparavant, qu'on l'intègre ou qu'on le rejette (...) toute connaissance, par le fait même qu'elle prend appui sur la prise en compte de "documents" antérieurs, leur lecture et leur exploitation, est individualisée ». Les savoirs à enseigner renvoient donc aux connaissances du professeur nécessaires dans l'enseignement, c'est-à-dire "à la quantité et à l'organisation de la connaissance per se dans l'esprit du professeur» (Shulman, 2007 : 104). Ce dernier distingue les domaines et les catégories de connaissance des contenus (content knowledge) en les différenciant en une connaissance disciplinaire du contenu (subject matter content knowledge), une connaissance pédagogique du contenu (pedagogical content knowledge) et une connaissance du curriculum (curricular knowledge). Il s'intéresse ainsi aux sources du savoir professoral en avançant que «l'ultime test de la compréhension repose sur l'habileté à transformer sa connaissance en enseignement » (ibid.: 111). La connaissance disciplinaire du contenu requiert d'aller au-delà des faits ou des concepts d'un domaine, elle requiert en plus la compréhension des structures de la discipline. La connaissance pédagogique englobe les aspects de la connaissance liés à son enseignabilité et la connaissance du curriculum englobe la connaissance des programmes élaborés pour l'enseignement des domaines et sujets particuliers à un niveau donné. L'ensemble de ces savoirs est mobilisé par les pra- 
tiques dans des situations, au travers d'actions qui s'organisent au sein de dispositifs pédagogiques.

En parallèle de cette épistémologie scolaire, différents auteurs ont défini l'épistémologie professionnelle des enseignants qui permet, à notre sens, d'éclairer la question de la maîtrise des savoirs professionnels pour les enseignants.

Cette notion développée au sein des sciences de l'éducation exprime, en suivant l'analogie avec l'épistémologie générale, un discours sur les savoirs professionnels. Elle est liée à une épistémologie largement issue de la pratique et utilisée dans la pratique, c'est en ce sens qu'elle est qualifiée d' "épistémologie pratique » ou «professionnelle » (Chevallard, 2003). La notion de savoirs de métier ou de savoirs professionnels nous interroge pour, d'une part, mieux comprendre son lien avec les savoirs de référence et, d'autre part, pour tenter de cerner sa place dans les savoirs à enseigner.

Les savoirs de métier sont un type de savoir global et singulier qui privilégie l'intelligence pratique nécessaire à l'action. Le primat est ici accordé à l'acquisition de méthodes, dans le sens où les méthodes sont caractéristiques du savoir scientifique, c'est-à-dire qu'elles mettent en œuvre une notion de causalité et de conséquence, visant donc à «comprendre pourquoi ». Les procédés techniques sont ainsi «naturalisés » par rapport aux tâches. Cette approche s'oppose à la perception de la théorie comme préalable à la pratique ce qui pose la question du fonctionnement des savoirs dans les techniques.

Si les savoirs scientifiques n'ont pas vocation à produire l'action, ils sont mobilisés dans la construction d'un savoir ayant des finalités pratiques. Si «le geste habile révèle souvent un savoir plus considérable qu'on ne le croit » (Schön, 1996 : 218), on est souvent incapable de décrire le savoir que révèle l'action, il s'agit alors d'un savoir tacite. La réussite d'une action constitue un savoir-faire mais il y a forcément un niveau de compréhension, de conceptualisation, d'abstraction. Il s'agit donc de travailler sur le «conscientisable », c'est-à-dire de retrouver une source d'information sur l'action, de la verbaliser. On peut ainsi parler de savoirs d'action, savoir parce que l'information dégagée ici a des propriétés de transmission, de mémorisation, de traitement mais dont la source vient de l'action. La mobilisation des savoirs scientifiques peut donc se repérer en fonction de la finalité poursuivie par l'acteur, en situation d'action, au travers des savoirs professionnels. La notion de savoirs professionnels est entendue au sens où celui qui y recourt est un bon professionnel capable de contrôler et d'anticiper. Ces savoirs font référence à un ensemble de gestes qui vont de soi. Ce sont donc les gestes d'une technique, qui ont été enseignés mais sans forcément que soit acquise la conscience de leur rattachement aux savoirs élémentaires. La question est alors de comprendre comment les savoirs professionnels s'articulent aux savoirs scientifiques. La spécificité des savoirs professionnels, irréductible à des savoirs techniques ou scientifiques, peut se caractériser par des savoirs de référence pluriels qu'il reste cependant à identifier clairement.

Les savoirs professionnels se situent au sein de savoirs de référence qui ne doivent pas cacher le risque de confusion ou de subordination d'un savoir à un autre, influencés par les lieux d'acquisition. En effet, les savoirs ne sont pas isolés mais ils se situent dans des rapports personnels ou institutionnels en relation avec la tâche pour laquelle ils sont mobilisés dans une situation donnée.

Ces notions d'épistémologie scolaire et d'épistémologie professionnelle offrent des descripteurs pertinents pour analyser le découpage et la référentialité des savoirs dans un enseignement professionnel tel qu'étudié ici. C'est donc à partir de ces éléments qu'un protocole méthodologique a été mis en place pour recueillir 


\section{REFERENTIALITE ET DECOUPAGE DES SA VOIRS \\ DANS L'ENSEIGNEMENT PROFESSIONNEL AGRICOLE}

des données quant à la manière dont les enseignants négocient les savoirs à enseigner en référence à différents types de savoirs dans les phases de préparation de séances au sein de leurs disciplines.

\section{CHOIX METHODOLOGIQUES}

\section{Contexte et méthode}

L'approche méthodologique développée articule deux parties : une analyse des savoirs à enseigner dans deux disciplines spécifiques de l'enseignement agricole (information-documentation et Sciences et techniques des agroéquipements (STEA) et une analyse des savoirs enseignés au travers de la préparation de deux séances dans chacune des deux disciplines. L'analyse des savoirs se base sur un corpus de référentiels de formation et sur l'enregistrement de préparations de cours entre binômes d'enseignants dans chacune des deux disciplines où nous proposons de repérer le découpage des savoirs et leur référentialité à partir d'une analyse de verbatims.

Précisons que l'information-documentation est introduite comme discipline au sein de modules pluridisciplinaires dans les programmes de formation dans l'enseignement agricole dès 1984.Notons qu'aucune heure élève en documentation n'apparaît dans les programmes des autres filières professionnelles (tertiaires ou industrielles). Vingt heures, prises en charge par les professeurs documentalistes, sont allouées à la documentation dans les programmes de baccalauréat professionnel agricole dans un module général nommé MG1 comprenant également le français, l'éducation socioculturelle et l'histoire-géographie. Le module dans lequel s'insère l'information-documentation en baccalauréat professionnel se nomme : Langue française, langages, éléments d'une culture humaniste et compréhension du monde. L'objectif général du module est de «mobiliser des éléments d'une culture humaniste pour se situer et s'impliquer dans son environnement social et culturel».

La discipline scolaire (STEA) est au carrefour de plusieurs disciplines scientifiques de référence : les techniques d'une part, avec la mécanique, l'hydraulique, l'électricité, l'automatisme... mais aussi en lien avec d'autres disciplines comme l'agronomie, la zootechnie, la physique, la chimie... Les prescriptions scolaires tentent d'être assez larges pour prendre en compte toutes les pratiques agricoles tributaires des climats, des types de sols, des cultures, pour adapter les équipements agricoles.

Les savoirs à enseigner étudiés en information-documentation se situent dans l'objectif 4 du module MG $1^{1}$. Les savoirs à enseigner étudiés en STEA se situent dans le module professionnel EP3 ${ }^{2}$.

La prescription définissant le savoir à enseigner dans les deux disciplines étudiées ici, reste très large, même dans le document d'accompagnement ${ }^{3}$, renvoyant ainsi une grande partie du travail de transposition aux enseignants euxmêmes. Autrement dit, la transposition externe laisse une large part à la transposi-

Référentiel de formation documentation baccalauréat professionnel disponible sur (consulté le 12 avril 2016) : http://cdi.enfa.fr/files/2014/07/bacpro-tc-MG12014.pdf

Référentiel de formation de seconde professionnelle «production » disponible sur (consulté le 12 avril 2016): http://www.chlorofil.fr/fileadmin/user_upload/diplomes/ref/2nde/PV/2nde-DA-EP3-PVAgroeq.pdf

Document d'accompagnement du référentiel de formation en documentation du baccalauréat professionnel disponible sur (consulté le 12 avril 2016) : http://cdi.enfa.fr/files/2014/07/BacPro-DAMG1_juin2015.pdf 
tion interne. Pour l'information-documentation, les savoirs sur l'information sont centrés explicitement sur les aspects «typologie» tout en faisant référence à des connaissances plus larges sur la notion elle-même. Un certain nombre d'implicites qui renvoient aux connaissances individuelles de chaque professeur-documentaliste sont présents dans ces référentiels. Les savoirs à enseigner sont clairement définis en référence à une discipline scientifique, ceux des sciences de l'information et de la communication, et décrivent de manière succincte des contenus de savoir qu'il reste à définir précisément pour les enseigner. Pour les STEA, la prescription se présente sous forme d'objectifs et de capacités et peu de références sont affichées en ce qui concerne les savoirs scientifiques et techniques sous-jacents. Dans ce module de STEA, les prescriptions sont assez «génériques » et comportent des savoirs variés sous-jacents à des capacités. Chaque objectif identifie cependant un savoir, mais qui n'est pas explicitement défini, et qui vise des capacités à atteindre devant permettre de répondre aux spécificités locales. Le document d'accompagnement précise les modalités pour atteindre ces objectifs tout en restant lui aussi laconique. Ainsi l'exemple de la notion d'équilibre tracteur-outil est décrit sans référence aux mécanismes ou principes inhérents à cette liaison ni aux situations professionnelles représentatives.

Présentation des séances pédagogiques préparées

Nous avons mis en place, dans le cadre de la formation des enseignants, un protocole de préparation et réalisation de séances entre pairs. La préparation de la séance à réaliser est faite en commun, puis chaque enseignant effectue cette séance pendant que l'autre l'observe, ce qui permet ensuite, dans des entretiens postséances, d'échanger sur les pratiques de chacun.

Les préparations de séances entre deux enseignants ont été filmées et retranscrites. Leur déroulement a également été filmé mais nous n'utiliserons pas ce matériau dans cet article, nous limitant à une réflexion sur les savoirs à enseigner, les savoirs enseignés feront l'objet d'autres analyses.

Comme on peut le voir dans le tableau 1, les deux enseignants en information-documentation ont préparé une séance sur le thème de l'information et les enseignants en STEA ont préparé une séance sur le thème de la liaison tracteursoutils.

\begin{tabular}{|l|l|l|l|l|}
\hline Séances & \multicolumn{1}{|c|}{ Thèmes } & Enseignants & Type de séance et lieu & \multicolumn{1}{c|}{ Classe } \\
\hline \multicolumn{3}{|l|}{ Information-documentation } \\
\hline 1 & L'information & E1 & $\begin{array}{l}\text { Cours et travaux dirigés } \\
\text { (TD) en classe }\end{array}$ & $\begin{array}{l}\text { Première baccalauréat } \\
\text { professionnel }\end{array}$ \\
\hline 2 & L'information & E2 & Cours et TD en classe & $\begin{array}{l}\text { Première baccalauréat } \\
\text { professionnel }\end{array}$ \\
\hline$S T E A$ & $\begin{array}{l}\text { Liaison tracteur- } \\
\text { outil }\end{array}$ & E3 & Cours TD en classe & Seconde professionnelle \\
\hline 3 & $\begin{array}{l}\text { Liaison tracteur- } \\
\text { outil }\end{array}$ & E4 & Cours TD en classe & Seconde professionnelle \\
\hline 4
\end{tabular}




\section{RESULTATS ET ANALYSE}

Analyse des savoirs à enseigner dans les référentiels de formation en information-documentation

Les objectifs d'enseignement en information-documentation en baccalauréat professionnel sont centrés sur le concept «information». La visée de cet enseignement est à la fois professionnelle et culturelle. En effet l'insertion dans un module pluridisciplinaire le place clairement au service d'une visée de compréhension du monde. Dans la prescription le concept «information » est décliné au travers principalement de sa typologie et de ses caractéristiques (durable, éphémère, utile). Or, d'un point de vue scientifique l'information «est une connaissance communiquée ou communicable, en d'autres termes le contenu cognitif d'une communication réalisée ou possible» (Meyriat, 1983: 68). L'information est tout à la fois du contenu et une relation intellectuelle mais dès que le contenu s'extériorise entre différentes personnes, il le fait à travers une forme, un médium, une réalité matérielle qui constitue un moyen de transfert, ce qui autorise à avancer la notion de "relativité de l'information à une situation» (Quéré, 2000 : 351). Elle est une connaissance construite et circulante dans des processus de communication, elle est utile, plus ou moins spécialisée et plus ou moins durable. Elle est inscrite sur un support, ce qui lui permet d'être véhiculée en s'affranchissant du temps et de l'espace. Une information est utile si elle permet d'agir physiquement ou intellectuellement c'est-à-dire qu'elle apporte une connaissance dont on avait besoin pour prendre une décision. Meyriat (1981: 60) oppose "l'information de renseignement » d'utilité immédiate, et «l'information d'utilité durable » qu'il définit comme la connaissance reçue qui "vient s'ajouter à d'autres qui avaient été conservées et dont l'ensemble structuré constitue un savoir qui s'enrichit cumulativement ». Il propose d'en caractériser le genre, à savoir le croisement entre sa fonction (être utile explicitement ou avoir une utilité diffuse, plaire, divertir) et sa durée de vie (instantanée ou durable et définitive). L'information peut ainsi être différenciée en fonction de son contexte d'énonciation, de sa structuration, de son canal de diffusion ou du niveau de son contenu. Elle peut donc être qualifiée d'information scientifique et technique (spécialisée), médiatique, culturelle ou de renseignement. Autrement dit la caractérisation de l'information telle que suggérée dans le référentiel ne reprend qu'une partie de sa définition scientifique, puisque pour établir une typologie de l'information il est nécessaire d'en caractériser le genre qui est scientifiquement défini comme le croisement de la durée de vie et de la fonction.

On perçoit donc à travers cet exemple d'objet de savoir à enseigner l'écart, la distance ou encore la dimension implicite entre la définition scientifique du concept et le savoir à enseigner tel qu'il est décrit. Pourtant c'est bien ce croisement qui permet de distinguer l'utilité de l'information dans un contexte professionnel.

Analyse des savoirs à enseigner dans les référentiels de formation en STEA

Les objectifs d'enseignement en STEA en seconde professionnelle sont centrés sur le concept «liaison tracteur-outil». Dans cette partie du référentiel cette notion doit être étudiée dans une visée professionnelle permettant à l'élève d'acquérir des capacités professionnelles. Dans ce cas, l'attelage d'un tracteur et d'un outil et la connaissance des différents éléments permettant de réaliser cette note 4 .

Rappelons que ces textes de référence sont disponibles sur Internet et que le lien est indiqué à la 
fonction sont recommandées. La référence aux savoirs savants de cette discipline scolaire est complexe car elle renvoie à plusieurs champs scientifiques et techniques et il n'existe pas une définition spécifique des savoirs savants de l'agroéquipement. Aussi, nous avons pris le parti de synthétiser dans cet article les références aux savoirs liés à la liaison tracteur-outil qui nous intéressent ici.

Ainsi, les attelages sont un ensemble de dispositifs de liaison entre le tracteur et les outils portés, semi-portés et trainés (CEMAGREF, 1991). Ce premier élément de savoir, invite donc à appréhender différents types d'outils avant de découvrir les différents éléments d'un attelage de tracteur permettant de réaliser une liaison tracteur-outil. De plus les organes du tracteur permettant de réaliser cette liaison sont à associer avec les différents types d'outils.

Donc cette prescription renvoie implicitement, tout d'abord, à la notion d'attelage mécanique du tracteur aux outils. Nous pouvons prendre comme exemple le cas des outils semi-portés, qui sont adaptés au tracteur de manière à ce que celui-ci supporte une partie du poids de l'outil et celui-ci possède au moins un point d'appui au sol. Le savoir sur ces outils semi-portés renvoie également à une pluralité de savoirs. En effet il s'agit là d'une notion d'équilibre statique de l'ensemble tracteur-outil qui elle-même se réfère à la notion de statique du solide en sciences physique. Ce type d'outil est associé au piton d'attelage du tracteur, axe vertical situé sur la partie basse et arrière du tracteur capable de soutenir des charges importantes et équipé d'une broche ou d'un verrou qui empêche l'anneau de l'outil de sortir. Cependant, la majorité de ce type d'outils a besoin d'une énergie électrique, hydraulique, pneumatique pour fonctionner et qui provient du tracteur. La liaison tracteur-outil renvoie donc d'une part à une opération permettant une liaison mécanique entre les deux et d'autre part à des liaisons électriques et/ou hydrauliques permettant d'assurer l'animation de l'outil. La prescription du référentiel bien qu'étant discrétionnaire (Gillet \& Fauré, 2014), renvoie donc à plusieurs notions inter-reliées qu'il s'agit de sélectionner et d'ordonner de manière à démarrer le processus de transposition didactique interne.

Mise au point des savoirs à enseigner

dans les préparations communes de séances pédagogiques

Préparation de la séance sur l'information

Deux enseignantes discutent dans cette phase de préparation commune sur la conception pédagogique de la séance :

«Ce que tu veux, c'est introduire la notion de durabilité alors du coup est-ce que ce tableau, comme si on était dans un diaporama où on montre de façon progressive, on conclut de ça uniquement la partie sur la fonction et après par rapport à une fonction, on introduit le critère de temps ? (E1) On amène le tableau en 2 temps ? » (E2). Cette proposition de découpage est reprise et précisée ensuite : «Pourquoi pas l'introduire en 2 temps, c'est possible mais en fait la question c'est aussi est-ce que pour qualifier, pour se positionner dans le tableau, on a besoin du pôle émission et du pôle réception? » (E2).

On voit que la concertation implique ici d'expliciter clairement les attendus qui relèvent souvent d'implicites, ce qui permet de préciser les liens entre situations pédagogiques et objets de savoirs à enseigner dans une forme de négociation des savoirs : "dans les situations proposées, on met comme préalable il faut qu'il $y$ ait une situation clairement orientée du côté de l'intentionnalité et clairement une situation orientée côté réception pour évaluer la durée de vie de l'information » $(\mathrm{E} 1)$. 


\section{REFERENTIALITE ET DECOUPAGE DES SA VOIRS DANS L'ENSEIGNEMENT PROFESSIONNEL AGRICOLE}

Cette phase de conception pédagogique se confronte également à des questions de découpage didactique renvoyant aux définitions même des objets de savoir à enseigner :

« et du coup dans un cas, elle peut être à durée de vie éphémère et dans l'autre elle peut être durable, dans ce cas-là il faut voir si ça marche » (E2). L'avancée se fait par tâtonnements progressifs : " on essaie, admettons que l'exemple on le prend, on fait l'émetteur, le prof, un cours, faire apprendre, ensuite on passe ici, voyons si ça marche, avant de passer au récepteur. On rajouterait ici, quelque chose qui ait du contenu, c'est à dire quel est le contenu, le cours sur les systèmes d'information, quelle est sa fonction, là on va se mettre d'accord pour l'exemple et que c'est durable, ensuite on regarde qui est récepteur»(E2). Le dialogue se poursuit pour se mettre d'accord afin de lever les ambiguités : «finalement c'est la situation... que ce ne soit pas ambigu là dans ce qu'on propose...» $(\mathrm{E} 1)$.

Ici la définition de la typologie de l'information est confrontée à un problème de maîtrise par les enseignants. On voit en effet un certain tâtonnement dans les choix à opérer qui renvoie à une difficulté d'appréhension scientifique. Par ailleurs, ces choix se révèlent encore plus complexes du fait du nécessaire découpage didactique. D'une part, séparer la fonction et la durée de vie pose problème mais ne pas les séparer est trop compliqué à mettre en œuvre dans un exercice, d'autre part, il parait difficile de pouvoir caractériser l'information au niveau durée de vie et fonction sans le rapprocher de la position énonciateur ou récepteur. Autrement dit, la complexité du savoir à enseigner se confronte ici clairement à la distance avec le savoir de référence et à sa maîtrise.

« Par exemple quand il s'agit de faire varier la situation de communication pour montrer que suivant le contexte il peut y avoir une information qui a une fonction différente et bah là des fois ce n'est pas évident, de la même façon que ce n'est pas évident de trouver des exemples en info doc pour introduire la complexité des savoirs qu'on est en train de parler» (E2). La discussion sur la complexité de la mise en œuvre d'un savoir avance en précisant le domaine de validité : «mais après tu sais aussi le fait de maitriser une notion pour le vérifier et peut-être proposer une solution de plus en plus complexe pour tester, si tu veux, la notion d'acquisition de la notion aussi donc en rester sur des trucs pratique documentaire, pourquoi pas ? Mais après le modèle il est pour la documentation mais il repose quand même sur une certaine utilité ailleurs » (E1).

L'enseignement en information-documentation situé dans un module pluridisciplinaire se doit de concourir au développement d'une culture de l'information re-mobilisable dans les autres disciplines. Ainsi la question de la caractérisation de l'information doit trouver sa légitimité en information-documentation et son utilité dans les autres disciplines.

Préparation de la séance sur la liaison tracteur-outils

Les deux enseignants débattent sur les contenus et objectifs de la séance : « on part sur les schémas de reconnaissance les trois modes d'attelage c'est ça ?» (E4). La première piste se confronte à l'imprécision : «non c'est plutôt identifier les travaux d'attelage»(E3), mais tend à se compléter en recherchant le consensus: «ou c'est ça compléter un schéma de reconnaissance des travaux de l'attelage et situer les emplacements des outils sur un tracteur $\gg(\mathrm{E} 4)$ ou encore : " pour dire qu'il peut y avoir des relevages avant ou arrière... identifier les différences », enfin : " oui tu as raison qu'ils repèrent qu'à l'avant il n'y a pas de chandelles, pas de rélevateur» (E3).

Ces premiers éléments de définition du contenu vont se poursuivre et s'affiner petit à petit sans que la référence scientifique soit convoquée d'emblée : «on a un tracteur avec un outil porté, il faut repérer les éléments du tracteur en liaison avec l'outil, pour identifier les éléments »(E4), avec un essai de repérage des 
éléments importants pour soi d'abord : « rôle du tracteur et rebondissement sur la partie relevage puis passage au dispositif d'attelage remorque »(E3). Cependant des éléments de plus en plus précis et techniques sont amenés : «spécificités des crochets tournants... La barre oscillante a une limite de charge verticale, $500 \mathrm{~kg}$ je crois, le crochet tournant a une limite de charge horizontale de 3 tonnes » (E4), ou encore : « introduire d'autres éléments d'attelage comme l'éclairage ou l'hydraulique » (E3), en prenant conscience des éléments laissés de côté : « on a pas rappelé que le relevage est actionné par le système hydraulique du tracteur »(E3), puis préciser encore : «La hauteur du relevage est variable grâce à un dispositif hydraulique depuis le poste de pilotage », «Actionné par la force hydraulique du tracteur via un dispositif de commande (E4).

Pour en venir finalement à une définition plus complète :

«il y a des outils portés, c'est des outils où le poids de l'outil est complètement sur le tracteur, rien qui repose au sol quand on est position de transport, en position de travail c'est différent puisque l'outil peut travailler dans le sol par exemple, les outils semi-portés quant à eux ont une partie seulement sur le tracteur l'autre partie est sur un essieu avec des roues sur l'outil, les outils traînés où il n'y a aucune charge verticale sur le tracteur, la seule charge qu'il y a c'est frein outil avec charge horizontale, essieu avant essieu arrière, les trois n'ont pas la même action sur le tracteur » (E3),

ce qui permet à l'autre enseignant d'enchaîner : « sur les dispositifs d'attelage sur remorque, il existe aussi des crochets rabatteurs, ramasseurs, des boules, des rotules»(E4), avec plusieurs allers-retours sur les éléments susceptibles d'intégrer le savoir à enseigner : « pour certaines machines il faut un relevoir, une animation à la prise force, un éclairage arrière, voire un attelage hydraulique, un accouplement hydraulique quoi »(E3). Puis nous trouvons un essai de synthèse permettant de se mettre d'accord et d'avoir une vision plus englobante : «Donc l'objectif c'est l'incidence du mode d'attelage sur le report de charge du tracteur » (E4), complétée immédiatement ainsi : «c'est la géométrie de l'attelage trois points, le raisonnement de l'ordre d'attelage et de dételage ainsi que les différents types d'attelage la chape, le piton et la barre oscillante » (E3).

Nous pouvons constater aussi des tâtonnements entre les deux enseignants avant d'arriver à choisir et à définir les objets de savoirs à enseigner sur un des objectifs du référentiel. Nous percevons des difficultés à se mettre d'accord sur une formulation précise et scientifique d'un objet de savoir à partir de définitions de capacités à acquérir. Ce qui entraîne aussi des difficultés de choix de situations pédagogiques où ces savoirs sont mis en œuvre.

\section{DISCUSSION ET CONCLUSION}

Ces données, bien que partielles, permettent d'avoir une première approche sur la compréhension de l'appropriation par les enseignants du découpage des savoirs en vue de leur enseignement et, par la même, nous offrent des indices quant à la référentialité de ces savoirs dans la phase d'apprêt didactique.

Nous retrouvons, par exemple, dans ces résultats la partition de Shulman (2007) sur les savoirs curriculaires, pédagogiques et de contenus disciplinaires. À ceux-là se rajoutent les savoirs professionnels visés. Dans les deux disciplines, les savoirs curriculaires renferment un certain nombre d'implicites et laissent à l'enseignant une grande latitude dans la transposition didactique interne.

L'épistémologie professionnelle, théorie de la connaissance enseignée (Amade-Escot, 1998), lorsque deux enseignants travaillent sur un même objet de savoir à enseigner, se révèle hésitante, parfois mouvante dans ses référentialités et ceci pour deux raisons apparentes dans nos résultats. D'une part, lorsque ceux-ci 
sont confrontés aux savoirs pédagogiques nous voyons que le savoir lié aux contenus disciplinaires a du mal à s'exprimer clairement et, d'autre part, la complexité croît quand ces deux types de savoirs sont mis en relation avec les savoirs professionnels visés.

L'épistémologie scolaire telle qu'elle se met en jeu dans un enseignement professionnel agricole réfère à une multitude de savoirs difficiles à mobiliser pour les enseignants dans leurs pratiques. En effet, les résultats mettent au jour une négociation des savoirs à enseigner qui montre une difficulté dans le partage des savoirs de référence pour les enseignants confrontés aux savoirs multiples au sein de ces disciplines scolaires.

L'enseignement agricole, en information-documentation et STEA, semble effectivement être confronté à une certaine complexité, inhérente à tout enseignement professionnel, quant aux savoirs à enseigner. En effet, dans ce type d'enseignement c'est l'acquisition à la fois de connaissances et de capacités qui est visée, ce qui semble renvoyer pour l'institution et pour les enseignants à des difficultés dans le découpage des savoirs et dans leur référentialité.

Cette complexité interroge ainsi l'identification, la sélection et le découpage des savoirs à enseigner, donc le processus de transposition didactique interne ainsi que les pratiques d'enseignement. Il existe un écart entre la figure du professionnel dessinée en filigrane dans les référentiels et celle réellement visée par les enseignants telle qu'elle se révèle dans les préparations communes de séances pédagogiques.

Les résultats montrent une dialectique entre savoirs pour le travail et savoirs académiques qui traversent les référentiels de formation. Cette tension entre utilité, contextualité et universalisme caractérise l'enseignement professionnel. Nous la retrouvons dans la composition des groupes qui élaborent les référentiels. Ceux-ci mixent en effet des représentants de l'institution, des enseignants, des formateurs d'enseignants et des représentants des corps professionnels concernés. Cette dialectique participe à la redéfinition du «professionnel » mais aussi au découpage des modules et des spécialités d'enseignants, comme nous l'avons vu avec l'information-documentation et les STEA. Cette particularité se retrouve dans les concours de recrutement des enseignants, la formation des enseignants mais aussi dans la composition des corps d'inspection. Ainsi, cette recherche, présentant ici un aspect exploratoire, ouvre une voie potentielle vers une analyse plus poussée des disciplines scolaires et des pratiques d'enseignement dans l'enseignement agricole.

\title{
Laurent FAURÉ Cécile GARDIÈS Jean-François MARCEL UMR EFTS ENFA
}

Université fédérale de Toulouse Midi Pyrénées

\begin{abstract}
The question of knowledge in education system arises several levels, including the referentiality of knowledge and his report by the different actors of the community. Agricultural education is «original» in the landscape of training in France because of the way knowledge is cut. Therefore, we analyze the impact of this particular division of knowledge for teachers. We study links between academic knowledge as defined by the institution and the issue of reference of this knowledge for teachers from a
\end{abstract}


theoretical framework articulating epistemology of knowledge and professional epistemology of the teachers and mobilizing a qualitative methodological approach. We analyse data collected and discuss how it is questioning teaching practices.

Keywords : agricultural education, epistemology of knowledge, professional epistemology, training reference, reference knowledge. 


\section{REFERENTIALITE ET DECOUPAGE DES SA VOIRS DANS L'ENSEIGNEMENT PROFESSIONNEL AGRICOLE}

\section{Bibliographie}

Amade-Escot C. (1998) «Apport des recherches didactiques à l'analyse de l'enseignement : une étude de cas, le contrat didactique »-in : C. Amade-Escot, J.-P. Barrué, J.-C. Bos, F. Dufor, M. Dugrand \& A. Terrise (éds.) Recherches en EPS : Bilan et Perspectives (253-260). Paris : Revue EP\&S

Arenilla L., Gossot B. \& Rolland M.-C. (1996) Dictionnaire de pédagogie. Paris : Bordas.

Arsac G., Chevallard Y., Martinand J.-L. \& Tiberghien A. (1994) La transposition didactique à l'épreuve. Grenoble : La Pensée Sauvage.

Beillerot J., Blanchard-Laville C. \& Mosconi N. (1996) Pour une clinique du rapport au savoir. Paris : L'Harmattan.

CEMAGREF, (1991) Lexique illustré du machinisme et des équipements agricoles. Paris : Tec \& Doc Lavoisier, Collection Formagri. Vol 1.

Chervel A. (1988) «L'histoire des disciplines scolaires, réflexions sur un domaine de recherche »- Histoire de l'Éducation 38 (59-119).

Chevallard Y. (1985/1991) La transposition didactique. Du savoir savant au savoir enseigné. La Pensée Sauvage: Grenoble.

Chevallard Y. (1996) «La fonction professorale : esquisse d'un modèle didactique »- in : Actes de la VIII École d'étude didactique des mathématiques (83-122). Clermont-Ferrand : IREM de Clermont-Ferrand

Chevallard Y. (2003) « Approche anthropologique du rapport au savoir et didactique des mathématiques »-in : S. Maury et M. Caillot (dirs.) Rapport au savoir et didactiques (81-122). Paris : Fabert.

Gardiès C. \& Hervé N. (dirs.) (2015) L'enseignement agricole, entre savoirs professionnels et savoirs scolaires. Les disciplines en questions. Dijon : Éducagri éditions.

Gillet G. \& Fauré L. (2014) « Sciences et techniques des agroéquipements : quelle didactique pour quelle discipline ?»- in : C. Gardies et N. Hervé (dir.) L'enseignement agricole entre savoirs professionnels et savoirs scolaire. Les disciplines en question (23-42). Dijon : Éducagri éditions.

Jeanneret Y. \& Ollivier B. (2004) «Introduction. Une discipline et l'université française» - Hermès, La Revue 1, 38 (13-18).

Losfeld G. (1990) «Sciences de l'information VS sciences de la communication : éléments pour un dialogue épistémologique »-in : Actes du Congrès INFORCOM 90 (24-26 mai 1990 ; La Baume les Aix) La recherche en information-communication : l'avenir (161-166). Aix-en-Provence : Université de Provence.

Margolinas C. (2014) «Connaissance et savoir. Concepts didactiques et perspectives sociologiques? »-RFP 188 (13-24).

Martinand J.-L. (1989) «Pratiques de référence, transposition didactique et savoirs professionnels en sciences techniques »-Les Sciences de l'Éducation - Pour l'Ėre Nouvelle 2 (23-29).

Meyriat J. (1981) «Document, documentation, documentologie » - Schéma et Schématisation 14 (51-63).

Meyriat J. (1983) « De la science de l'information aux métiers de l'information » - Schéma et Schématisation 19 (65-74). 
Quéré L. (2000) «Au juste, qu'est-ce que l'information?»-Réseaux 18 (100) (331-357).

Schön D. A. (1996) «A la recherche d'une nouvelle épistémologie de la pratique et de ce qu'elle implique pour l'éducation des adultes »- in : J.-M. Barbier (dir.) Savoirs théoriques et savoirs d'action (201-222). Paris : PUF.

Shulman L. S. (2007) «Ceux qui comprennent, le développement de la connaissance dans l'enseignement »-Éducation \& Didactique Vol. 1 (97114). 